\title{
Minimal Detectable Biases of GPS observations for a weighted ionosphere
}

\author{
K. de Jong and P. J. G. Teunissen \\ Department of Mathematical Geodesy and Positioning, Delft University of Technology, Thijsseweg 11, 2629 JA Delft, The Netherlands
}

(Received November 25, 1999; Revised May 19, 2000; Accepted May 19, 2000)

\begin{abstract}
The theory and application of statistical quality control is well established in precise positioning, navigation and geodesy. Quality control is made up of several contributing factors, one of which is internal reliability. Internal reliability describes the ability to find biases in observational data and is represented by the Minimal Detectable Bias (MDB). The MDB provides a diagnostic tool to infer the strength with which positioning models can be validated. In this contribution closed-form expressions will be given for the MDBs of GPS code and carrier observations for three different baseline models: the geometry-free model and two variants of the geometry-based model. These expressions apply to any number of carrier frequencies. The expressions take into account the presence of ionospheric disturbances by weighting these effects. As such, they are applicable to baselines of any length.
\end{abstract}

\section{Introduction}

Minimal Detectable Biases (MDBs) as introduced by Baarda $(1967,1968)$ are important diagnostic tools for inferring the strength of model validation. They are said to describe the internal reliability of a system. MDBs can also be used to study the strength of the various GPS positioning models (single receiver, baseline and network). In de Jong (1999) analytic expressions are given for the MDBs of outliers and cycle slips in GPS code and carrier observations for a single baseline. In deriving these expressions, it was assumed that ionospheric effects may not always be eliminated when differencing between receivers. Since the influence of the ionosphere will increase with increasing baseline length, an ionospheric weighting factor was introduced to account for the ionospheric effects. Setting this factor to zero corresponds to the short baseline case. The MDBs for short baselines were already derived in Teunissen (1998). In this paper, simplified expressions, which are easy to implement, will be given for the code and carrier MDBs for three different baseline models. These expressions are valid not only for single- and dual-frequency data, but for any number of carrier frequencies.

In Section 2 a brief review is given of the concept of internal reliability. Section 3 gives a summary of the measurement models for the three single-baseline models, introduced in Teunissen (1998), together with their stochastic model. In the last two sections the simplified expressions for the MDBs of code and carrier observations are given.

\section{Internal Reliability}

Internal reliability, as represented by the MDBs, describes the size of the model errors that can just be detected using the appropriate test statistics. For more details, the reader is referred to, for example, Baarda (1968) or Teunissen (1985).

Copy right (C) The Society of Geomagnetism and Earth, Planetary and Space Sciences (SGEPSS); The Seismological Society of Japan; The Volcanological Society of Japan; The Geodetic Society of Japan; The Japanese Society for Planetary Sciences.
The null hypothesis $H_{0}$ describes the case model errors are absent. The alternative hypothesis $H_{a}$ considered here assumes there is a bias in one of the observations. These two hypotheses are defined as

$$
\begin{aligned}
& H_{0}: E\{y\}=A x, \quad D\{y\}=Q_{y}, \\
& H_{a}: E\{y\}=A x+c \nabla, \quad D\{y\}=Q_{y},
\end{aligned}
$$

where $E\{$.$\} and D\{$.$\} are the expectation and dispersion op-$ erators, respectively, $y$ the $m$-vector of observations, $x$ the $n$-vector of unknown parameters, $A$ the $m \times n$ design matrix, $c$ a known $m$-vector, which specifies the type of model error, and $\nabla$ its unknown size. The uniformly most powerful test statistic for testing $H_{0}$ against $H_{a}$ is given as

$$
T=\frac{c^{T} Q_{y}^{-1} P_{A}^{\perp} y}{c^{T} Q_{y}^{-1} P_{A}^{\perp} c}
$$

where the projector $P_{A}^{\perp}$ is defined as

$$
P_{A}^{\perp}=I-A\left(A^{T} Q_{y}^{-1} A\right)^{-1} A^{T} Q_{y}^{-1} .
$$

The test statistic $T$ has a Chi-squared distribution, which is central under $H_{0}$ and non-central under $H_{a}$. The noncentrality parameter $\lambda_{0}$ is a measure of the distance between $H_{0}$ and $H_{a}$. This non-centrality parameter can be computed once reference values are chosen for the level of significance (the probability of rejecting $H_{0}$ when it is true) and the detection power (the probability of rejecting $H_{0}$ when $H_{a}$ is true). Once the parameter is known, the corresponding size of the bias that can just be detected is given as

$$
|\nabla|=\sqrt{\frac{\lambda_{0}}{c^{T} Q_{y}^{-1} P_{A}^{\perp} c}} .
$$

This is the Minimal Detectable Bias. For most practical applications, $\alpha_{0}=0.001$ and $\gamma_{0}=0.80$, resulting in a noncentrality parameter $\lambda_{0}=17$. As can be seen from (5), 
the MDB not only depends on $\alpha_{0}$ and $\gamma_{0}$, but also on the functional and stochastic model, through the design matrix $A$ and the covariance matrix $Q_{y}$, and the alternative hypothesis considered, as represented by the vector $c$. The alternative hypotheses considered here consist of outliers and cycle slips in GPS code and carrier observations, respectively.

\section{Baseline Models}

The double difference (DD) measurement model for two receivers each tracking the satellites $r$ and $s$ at an epoch $t$, can be written as

$$
\begin{aligned}
p_{i}^{r s}(t)= & \rho^{r s}(t)+\mu_{i} I^{r s}(t)+n_{p_{i}}^{r s}(t), \\
\phi_{i}^{r s}(t)= & \rho^{r s}(t)-\mu_{i} I^{r s}(t)+\lambda_{i} N_{i}^{r s}+n_{\phi_{i}}^{r s}(t) \\
& i=1 \cdots f
\end{aligned}
$$

where $f$ is the number of frequencies, $p_{i}^{r s}$ and $\phi_{i}^{r s}$ are the DD code and carrier observations, expressed in meters, $\rho^{r s}$ the unknown satellite-receiver DD range, $N_{i}^{r s}$ the DD carrier ambiguities, $\lambda_{i}$ the carrier wavelengths, $\mu_{i}=\left(\lambda_{i} / \lambda_{1}\right)^{2}$, $I^{r s}$ the DD ionospheric effect and $n_{p_{i}}^{r s}$ and $n_{\phi_{i}}^{r s}$ the DD measurement noise of code and carrier. It is assumed here that tropospheric effects are either absent or accounted for using the appropriate models. Introducing the ionospheric pseudoobservable $I_{P}$, with sample values taken e.g. from an external ionospheric model, the ionospheric parameter can be eliminated from $(6)$, resulting in

$$
\begin{aligned}
p_{i}^{r s}(t)^{\prime}= & p_{i}^{r s}(t)-\mu_{i} I_{P}^{r s}(t) \\
= & \rho^{r s}(t)+n_{p_{i}^{\prime}}^{r s}(t), \\
\phi_{i}^{r s}(t)^{\prime}= & \phi_{i}^{r s}(t)+\mu_{i} I_{P}^{r s}(t) \\
= & \rho^{r s}(t)+\lambda_{i} N_{i}^{r s}+n_{\phi_{i}^{\prime}}^{r s}(t) \\
& i=1 \cdots f
\end{aligned}
$$

Using (7) the measurement model can be formulated for the three baseline models, considered here.

Geometry-free. For the geometry-free (GF) model the observation equations remain parametrized in terms of the unknown DD receiver-satellite ranges. As a result, they remain linear and the receiver-satellite geometry is not explicitly present in the measurement model. This means that the receivers may either be stationary or moving. This model has been studied in particular for carrier phase ambiguity resolution (Euler and Goad, 1991; Teunissen, 1996; Jonkman, 1998), and validation of GPS code and carrier observations (de Jong, 1996, 1997, 1998).

Thus, when $m$ satellites are tracked, there are $2(m-1)$ DD measurements per frequency for each epoch. The redundancy of the model equals $(m-1)((2 f-1) k-f)$, where $k$ denotes the number of observation epochs. In order to have redundancy, at least two satellites should be observed and the number of epochs $k$ should be greater than $f /(2 f-1)$. For $f=1$, this means $k$ should be greater than one, for all other (multi-frequency) cases, $k$ should at least be equal to one.

Roving receiver. For the roving receiver (RR) model, one receiver is stationary, whereas the other one is moving. The DD observation equations are parametrized in terms of the unknown baseline components. For each observation epoch, a new baseline is introduced. The RR model is a geometrybased model, since the receiver-satellite geometry appears in the observation equations through the linearized (in terms of the baseline components) DD ranges. For a single epoch the linearized expression for $\rho(t)$ (containing the DD ranges for $m-1$ satellite pairs) is given by

$$
\Delta \rho(t)=\rho(t)-\rho(t)_{0}=\bar{G}(t) \Delta b(t)
$$

where $\rho(t)_{0}$ denotes the DD range, computed at some initial value, $\Delta b(t)$ the corrections to the initial baseline vector $b(t)_{0}$ at epoch $t$ and $\bar{G}(t)$ the $(m-1) \times 3$ DD design matrix, which takes into account the relative receiver-satellite geometry. This geometry changes only slowly with time, due to the high altitude of the GPS satellites. In our further analysis we will consider only short observation time spans. Therefore $\bar{G}(t)$ will be assumed time-invariant, i.e., $\bar{G}(t)=\bar{G}$ for all $k$ epochs.

The redundancy for this model equals $f(m-1)(2 k-$ 1) $-3 k$. For the baseline components to be estimable, the minimum value of $m$ is four. If $k=1$ and $f=1$, redundancy exists if $m>4$; for all other cases, $m$ should at least be equal to four.

Stationary receiver. This is also a geometry-based model. For the stationary receiver (SR) model, both receivers are stationary. The DD observation equations are again parametrized in terms of the baseline components. For this model, the baseline is the same for all observation epochs. As a consequence, the redundancy, compared to the roving receiver model, is increased by $3(k-1)$ and equal to $f(m-1)(2 k-1)-3$.

It follows from (5) that in order to compute MDBs, the stochastic model of the observations is required. In Teunissen (1998) and de Jong (1999) the MDBs are derived for a very general model, which allows for correlation between observations and a different precision for each frequency. Here we will consider only a very simple stochastic model, which is widely used in practice.

The stochastic model for the single-differenced code, carrier and ionospheric observations to a particular satellite $s$, is assumed to be given by

$$
\begin{aligned}
& C_{p \phi}^{s}=w_{s} \cdot \operatorname{diag}\left(c_{p}^{2} I_{f} c_{\phi}^{2} I_{f}\right) \\
& \sigma_{I_{P}^{s}}^{2}=w_{s} \cdot s^{2}
\end{aligned}
$$

where $w_{s}$ is a satellite-dependent weighting factor and $I_{f}$ the $f \times f$ identity matrix. Through the weighting factor it is possible to assign different weights to each satellite, for example, depending on their elevation. In that case the weighting factor becomes time-dependent. However, as was done for the geometry, for short observation time spans it is taken as a constant.

Eliminating the ionospheric parameters using the observable $I_{P}$ results in the covariance matrix $C_{p \phi I}^{s}$ of the observations

$$
\begin{aligned}
C_{p \phi I}^{s}= & w_{s} \cdot\left(\left(\begin{array}{ll}
c_{p}^{2} I_{f} & \\
& c_{\phi}^{2} I_{f}
\end{array}\right)\right. \\
& \left.+s^{2}\left(\begin{array}{c}
\mu \\
-\mu
\end{array}\right)\left(\begin{array}{c}
\mu \\
-\mu
\end{array}\right)\right)
\end{aligned}
$$


where $\mu=\left(\mu_{1} \ldots \mu_{f}\right)^{T}$. If the ionosphere is absent or assumed known, $s^{2}=0$; if the ionospheric behavior is completely unknown, $s^{2} \rightarrow \infty$. Adding a pseudo-observable with infinite variance is equivalent to introducing an additional parameter. Therefore, the redundancy of each of the three baseline models decreases by $k(m-1)$. The two extreme cases are generally referred to as ionosphere fixed and ionosphere float. In practice $s^{2}$ may often vary between these two extreme values, depending on the baseline length (Schaffrin and Bock, 1988).

\section{Code Outlier MDBs}

The MDBs for outliers in code data will be given, based on the derivations in de Jong (1999) and Teunissen (1998) for the single difference (SD) observables. The carrier-tocode variance ratio, which in practice is of the order of $10^{-4}$, can be neglected here. Together with the assumptions of constant receiver-satellite geometry and constant weighting factors, this results in relatively simple expressions.

For an outlier at epoch $l, 1 \leq l \leq k$, in the SD code observable $p_{r}, r=1, \ldots, f$, to satellite $i \in\{1, \ldots, m\}$, we get for the geometry-free model

$$
\begin{aligned}
\left|\nabla_{p_{r}^{i}}\right|=\sigma_{p^{i}} & \left\{\lambda_{0} /\left[\left\{1-\frac{1}{k}\left[F_{1}\left(\mu_{r}\right) F_{2}\left(c_{p}^{2}\right)\right.\right.\right.\right. \\
& \left.\left.\left.\left.+F_{3}\left(\mu_{r}\right)\right]\right\}\left(1-\frac{w_{i}}{\sum_{j=1}^{m} w_{j}}\right)\right]\right\}^{1 / 2}
\end{aligned}
$$

and for the roving receiver model

$$
\begin{aligned}
\left|\nabla_{p_{r}^{i}}\right|=\sigma_{p^{i}}\{ & \lambda_{0}\left[\left\{1-\frac{1}{k}\left[F_{1}\left(\mu_{r}\right) F_{2}\left(c_{p}^{2}\right)\right.\right.\right. \\
& \left.\left.\left.\left.+F_{3}\left(\mu_{r}\right)\right]\right\}\left(1-\frac{w_{i}}{\sum_{j=1}^{m} w_{j}}\right)\right]\right\}^{1 / 2} .
\end{aligned}
$$

Within the approximations used, the code outlier MDBs for the roving and stationary receiver models are the same. The quantities that appear in (11) and (12) are defined as

$$
\begin{aligned}
F_{1}(x)= & s^{2} x /\left(c_{\phi}^{2}+s^{2} \sum_{j=1}^{f} \mu_{j}^{2}\right), \\
F_{2}(x)= & \left(1+x /\left(s^{2} \sum_{j=1}^{f} \mu_{j}^{2}\right)\right)^{-1}, \\
F_{3}(x)= & \left(c_{p}^{2}+s^{2} \sum_{j=1}^{f} \mu_{j}\left(\mu_{j}-x\right)\right)^{2} \\
& /\left\{f \cdot\left(c_{p}^{2}+s^{2} \sum_{j=1}^{f} \mu_{j}^{2}\right) .\right. \\
& \left.\left(c_{p}^{2}+s^{2} \sum_{j=1}^{f}\left(\mu_{j}-\bar{\mu}\right)^{2}\right)\right\}, \\
\bar{\mu}= & \frac{1}{f} \sum_{j=1}^{f} \mu_{j}, \\
P_{\left[G e_{m}\right]=} & \left(G e_{m}\right)\left[\left(G e_{m}\right)^{T}\left(G e_{m}\right)\right]^{-1}\left(G e_{m}\right)^{T},
\end{aligned}
$$

where $\left(G e_{m}\right)$ is the $m \times 4$ single difference design matrix. If $D^{T}$ is the $(m-1) \mathrm{x} m$ matrix which transforms single into

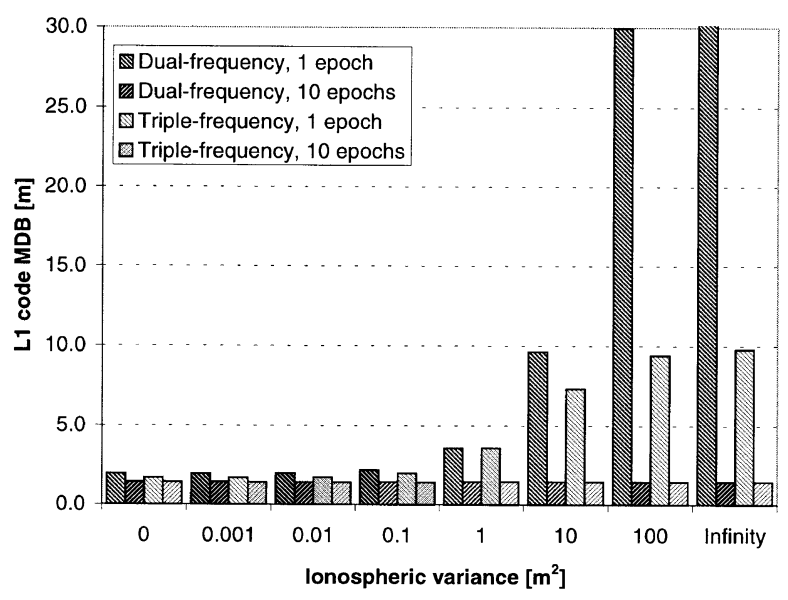

Fig. 1. Dual- and triple-frequency geometry-free L1 code MDBs as function of ionospheric variance; number of satellites is equal to five.

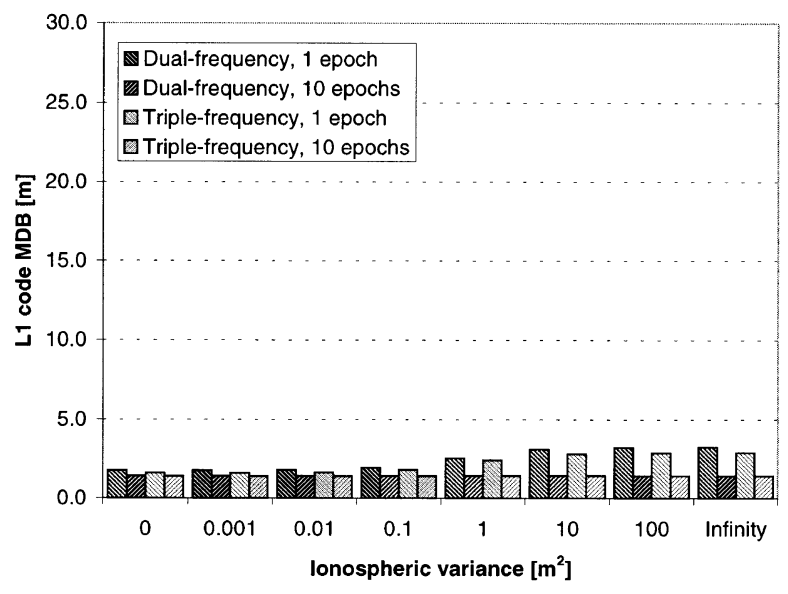

Fig. 2. Dual- and triple-frequency geometry-based L1 code MDBs as function of ionospheric variance; number of satellites is equal to five.

double differences, then $\bar{G}=D^{T} G$ and $D^{T} e_{m}=0$. The $m$-vector $e_{m}$ has all ones as its entries. If all satellites are assigned the same weight, we get

$$
1-w_{i} / \sum_{j=1}^{m} w_{j}=1-1 / m
$$

a situation which is assumed by most software packages developed for processing GPS data. In that case the MDBs for all satellites, for a particular frequency and baseline model, are the same.

If $m$, the number of tracked satellites, equals four, the design matrix $\left(G e_{m}\right)$ is a square matrix and the projector $P_{\left[G e_{m}\right]}$ reduces to the identity matrix. As a result, the term $1-c_{i}^{T} P_{\left[G e_{m}\right]} c_{i}$ becomes zero and the MDBs for all three baseline models become the same.

A further approximation is possible by realizing that $c_{i}^{T} P_{\left[G e_{m}\right]} c_{i}$ is the $i$-th diagonal element of the projector $P_{\left[G e_{m}\right]}$ and that the trace of this matrix is equal to its rank, which is four. The average value of the diagonal elements of this $m \mathrm{x} m$ matrix is therefore equal to $4 / m$, resulting in an average value of $1-c_{i}^{T} P_{\left[G e_{m}\right]} c_{i}=(m-4) / m$. 


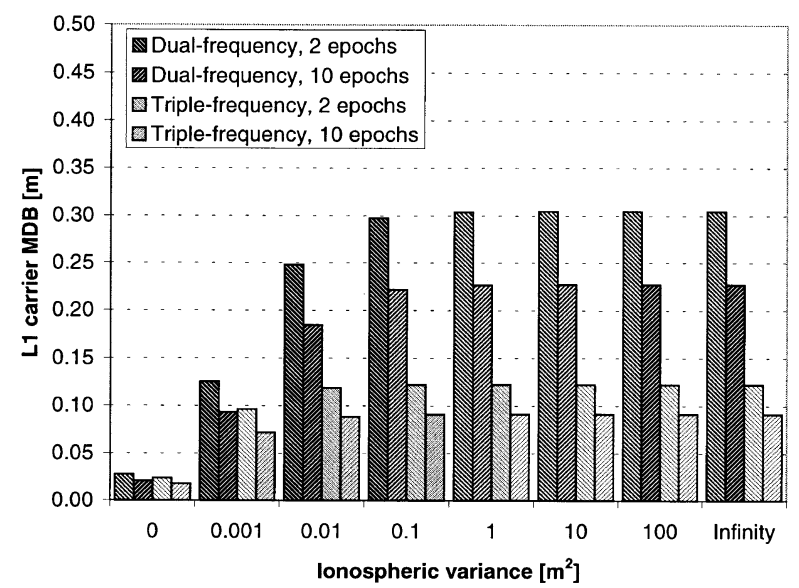

Fig. 3. Dual- and triple-frequency geometry-free L1 carrier MDBs as function of ionospheric variance and for a slip window of one epoch; number of satellites is equal to five.

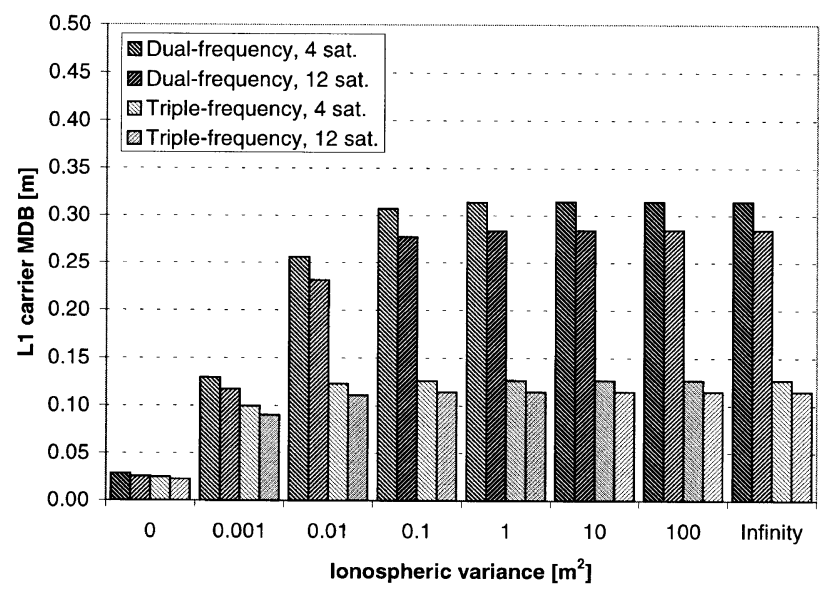

Fig. 4. Dual- and triple-frequency geometry-free L1 carrier MDBs as function of ionospheric variance and for a slip window of one epoch; number of epochs is equal to two.

Based on these simplifying assumptions, dual- and triplefrequency geometry-free and geometry-based code outlier MDBs were computed as a function of the ionospheric variance for $k=1$ and $k=10$. They are shown in Fig. 1 and 2 . For these and all other examples that follow, the noncentrality parameter $\lambda_{0}$ was set to 17 and the single difference standard deviations of code and carrier to $0.3 \mathrm{~m}$ and $0.003 \mathrm{~m}$, respectively. For the dual-frequency geometry-free ionosphere float case there is no redundancy and as a consequence the MDB becomes infinite. Comparing the MDBs of Fig. 1 and 2 we may conclude that when the number of epochs increases, the dual- and triple-frequency MDBs for both baseline models become more or less the same. In other words, the number of epochs becomes the main contributing factor to the redundancy.

\section{Carrier Slip MDBs}

Carrier slip MDBs will be expressed in units of range rather than in units of cycles. Where appropriate, the carrier-to-

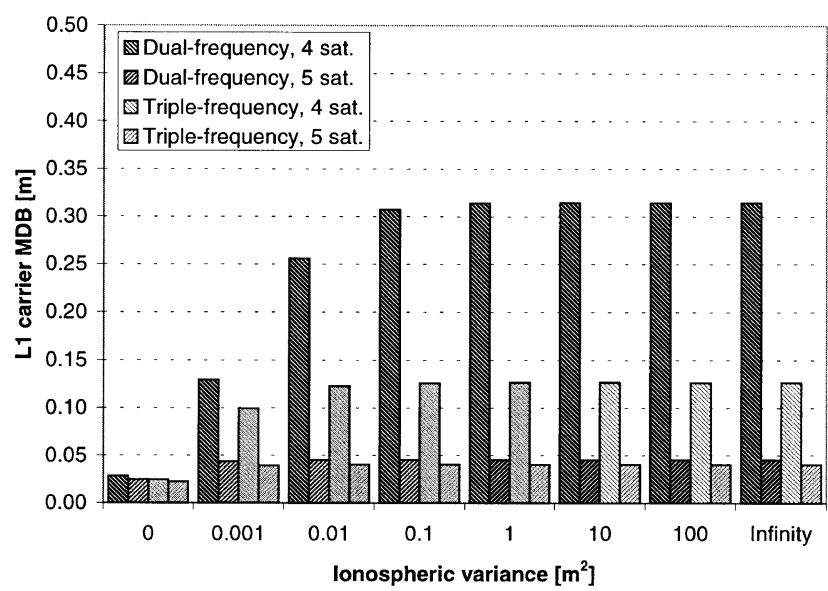

Fig. 5. Dual- and triple-frequency roving-receiver L1 carrier MDBs as function of ionospheric variance and for a slip window of one epoch; number of epochs is equal to two.

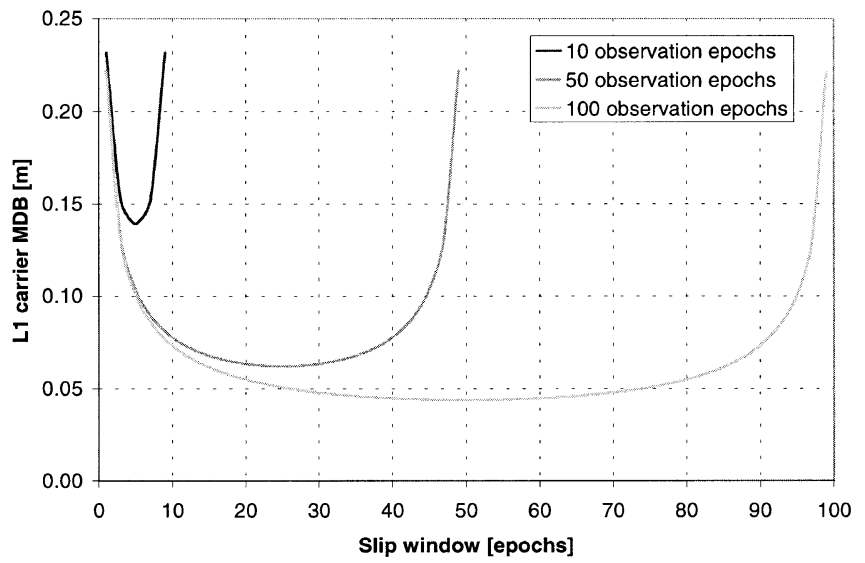

Fig. 6. Dual-frequency geometry-free ionosphere float L1 carrier MDBs as function of number of observation epochs and size of slip window; number of satellites is equal to four.

code variance ratio will be ignored. For a slip at epoch $l$, $1 \leq l \leq k$, in the SD carrier observable $\phi_{r}, r=1, \ldots, f$, to satellite $i \in\{1, \ldots, m\}$, we get for the three baseline models (geometry-free, roving receiver and stationary receiver, respectively)

$$
\begin{aligned}
\left|\nabla_{\phi_{r}^{i}}\right|=\frac{\sigma_{\phi^{i}}}{\sqrt{N}}\left\{\lambda_{0} /\left[\left(1-\frac{N}{k}\right)\left(1-\frac{w_{i}}{\sum_{j=1}^{m} w_{j}}\right) .\right.\right. \\
\left.\left.\left\{1-F_{1}\left(\mu_{r}\right)-F_{4}\left(\mu_{r}\right)\right\}\right]\right\}^{1 / 2}, \\
\left|\nabla_{\phi_{r}^{i}}\right|=\frac{\sigma_{\phi^{i}}}{\sqrt{N}}\left\{\lambda_{0} /\left[( 1 - \frac { N } { k } ) \left\{\left(1-F_{1}\left(\mu_{r}\right)\right.\right.\right.\right. \\
\left.-\frac{F_{4}\left(\mu_{r}\right)}{F_{5}\left(\mu_{r}\right)}\right)\left(1-\frac{w_{i}}{\sum_{j=1}^{m} w_{j}}\right) \\
\left.\left.\left.+\frac{F_{4}\left(\mu_{r}\right)}{F_{5}\left(\mu_{r}\right)}\left(1-c_{i}^{T} P_{\left[G e_{m}\right]} c_{i}\right)\right\}\right]\right\}^{1 / 2},
\end{aligned}
$$




$$
\begin{aligned}
&\left|\nabla_{\phi_{r}^{i}}\right|=\frac{\sigma_{\phi^{i}}}{\sqrt{N}}\left\{\lambda_{0} /\left[\left(1-\frac{N}{k}\right)\left(1-\frac{w_{i}}{\sum_{j=1}^{m} w_{j}}\right) .\right.\right. \\
&\left.\left.\left(1-F_{1}\left(\mu_{r}\right)\right)\right]\right\}^{1 / 2}
\end{aligned}
$$

where $N$ is the slip window, i.e., the period for which the slip is assumed present in the data, defined as $N=k-l+1$. Functions $F_{4}$ and $F_{5}$ are defined as

$$
\begin{aligned}
F_{4}(x)=\left(c_{\phi^{2}}+\right. & s^{2}\left\{(1+\varepsilon) \sum_{j=1}^{f} \mu_{j}^{2}\right. \\
& \left.\left.-(1-\varepsilon) x \sum_{j=1}^{f} \mu_{j}\right\}\right)^{2}, \\
F_{5}(x)= & \left(c_{\phi}^{2}+s^{2} \sum_{j=1}^{f} \mu_{j}^{2}\right) \\
& \left(f \cdot(1+\varepsilon) \cdot\left\{c_{\phi}^{2}+s^{2}(1+\varepsilon) \sum_{j=1}^{f} \mu_{j}^{2}\right\}\right. \\
- & \left.s^{2}(1-\varepsilon)^{2}\left(\sum_{j=1}^{f} \mu_{j}\right)^{2}\right)
\end{aligned}
$$

where $\varepsilon$ is the carrier-to-code variance ratio. It appears that this ratio is significant only for the geometry-free baseline model for large values of $s^{2}$; for the geometry-based models, it can be ignored.

Note that if $N=k$ the carrier slip MDBs become infinite. In this case a slip cannot (and does not have to) be separated from the carrier ambiguity. As a consequence, carrier MDBs can only be computed for $1<k<N$.

Unlike the code outlier MDBs, the carrier slip MDBs for the roving and stationary receiver are not the same. Like the geometry-free carrier MDBs, the stationary receiver carrier MDBs are independent of the receiver-satellite geometry. The geometry-free MDBs are larger than the stationary receiver MDBs, since $F_{4}\left(\mu_{r}\right) / F_{5}\left(\mu_{r}\right)>0$. The roving receiver MDBs are in between those of the geometry-free and stationary receiver models. In this case additional redundancy makes a difference with regard to internal reliability. If the number of satellites $m$ is equal to four, the geometryfree and roving receiver MDBs become the same.

Shown in Fig. 3 are the dual- and triple-frequency L1 carrier MDBs, again as a function of the ionospheric variance, for $k=2$ and $k=10$. The slip window $N$ was set to one. Even for the dual-frequency geometry-free ionosphere float model there is redundancy when $k=2$ and the MDBs are finite. However, the dual-frequency carrier MDBs increase rapidly to over one cycle with increasing ionospheric variance. For the triple-frequency case, however, all MDBs remain well below the single cycle level.

The geometry-free carrier MDBs were computed again, this time not as a function of the number of epochs, but as a function of the number of satellites. The results are shown in Fig. 4, from which we may conclude that the dualfrequency geometry-free carrier MDBs are of the same order of magnitude as those of Fig 3. For comparison, the roving receiver L1 carrier MDBs are shown in Fig 5. As already mentioned, when the number of satellites is equal to four, the geometry-free and roving-receiver MDBs are the same. When, however, the number of satellites is increased from four to five, we see a significant decrease in the size of the MDBs. This decrease may be attributed to the influence of the receiver-satellite geometry. The stationary-receiver carrier MDBs, not shown here, are of the order of a few $\mathrm{cm}$ and are hardly affected by the number of frequencies, the number of satellites and the ionospheric variance.

It is possible to decrease the size of the dual-frequency geometry-free carrier MDBs to below the single cycle level. This is accomplished by increasing both the number of epochs $k$ and the size of the slip window $N$. The dualfrequency geometry-free ionosphere float carrier MDBs for four satellites as a function of these two parameters are shown in Fig 6. The MDBs are symmetric around $k / 2$ and are already of the order of $0.15 \mathrm{~m}$ for $k=10$ and $N=3$ or $N=7$.

\section{Conclusions}

Expressions were given for code and carrier outlier MDBs for three different baseline models, which apply to any number of carrier frequencies. The expressions are valid for baselines of any length, since ionospheric disturbances are taken into account by weighting these effects.

The specific cases considered here applied to dual- and triple-frequency data. If ionospheric effects cannot be ignored, adding a third frequency is less important for reducing the size of the code MDBs than increasing the number of epochs. Already when the number of epochs is equal to ten, the ionosphere float MDBs are virtually the same as their ionosphere fixed counterparts for all dual- and triplefrequency baseline models.

For the geometry-free carrier MDBs adding a third frequency does make a significant difference for longer baselines in case the slip window is equal to just a single epoch. For dual-frequency observations the MDBs are always greater than one cycle, whereas for the triple-frequency case they are well below the single cycle level. The only way to bring the dual-frequency geometry-free MDBs below this level is by extending the slip window and increasing the number of epochs. For the geometry-based models, the carrier MDBs are much smaller than one cycle for both dual- and triple-frequency observations as long as the number of satellites is greater than four. Thus, if the proper observation scenario, which depends on the number of frequencies, satellites and observation epochs and the size of the slip window, is chosen, it is always possible to find even the smallest cycle slip.

\section{References}

Baarda, W., Statistical Concepts in Geodesy, Netherlands Geodetic Commission, Publications on Geodesy, New Series, vol. 2, no. 4, 74 pp., 1967.

Baarda, W., A testing procedure for use in geodetic networks, Netherlands Geodetic Commission, Publications on Geodesy, New Series, vol. 2, no. 5, 97 pp., 1968.

Euler, H. J. and C. Goad, On optimal filtering of GPS dual-frequency observations without using orbit information, Bulletin Géodésique, $\mathbf{6 5}$, pp. 130-143, 1991.

de Jong, C. D., Real-time integrity monitoring of dual-frequency GPS observations for a single receiver, Acta Geodaetica et Geophysica Hungarica, 31, pp. 37-46, 1996. 
de Jong, C. D., Principles and Applications of Permanent GPS Arrays, 105 pp., Delft University Press, Delft, 1997.

de Jong, C. D., A unified approach to real-time integrity monitoring of singleand dual frequency GPS and Glonass observations, Acta Geodaetica et Geophysica Hungarica, 33, pp. 247-257, 1998.

de Jong, C. D., Reliability of GPS observations using a weighted ionosphere, 1999 (in preparation).

Jonkman, N. F., Integer GPS Ambiguity Resolution without the Receiversatellite Geometry, LGR-series, no. 18, 95 pp., Delft Geodetic Computing Centre, Delft, 1998.

Schaffrin, B. and Y. Bock, A unified scheme for processing GPS dual-band phase observations, Bulletine Géodésique, 62, pp. 142-160, 1988.

Teunissen, P. J. G., Quality control in geodetic networks, in Optimization and Design of Geodetic Networks, edited by E. W. Grafarend and F. Sanso, pp. 526-547, Springer, Berlin Heidelberg New York, 1985.

Teunissen, P. J. G., An analytical study of ambiguity decorrelation using dual-frequency code and carrier phase, J. Geod., 70, pp. 515-528, 1996. Teunissen, P. J. G., Minimal Detectable Biases of GPS data, J. Geod., 72 pp. 236-244, 1998.

K. de Jong (e-mail: k.dejong@geo.tudelft.nl), P. J. G. Teunissen 